Science, Technology and Development 35 (3): 117-122, 2016

ISSN 0254-6418 / DOI: 10.3923/std.2016.117.122

(C) 2016 Pakistan Council for Science and Technology

\title{
Response of Strawberry (Frageria annanasa) cv. Chandler to Different Mulching Materials
}

\author{
${ }^{1}$ Muhammad Sudheer Tariq, ${ }^{1}$ Asghari Bano and ${ }^{2}$ Khalid Mahmood Qureshi \\ ${ }^{1}$ Department of Biological Sciences, Quaid-I-Azam University, Islamabad, Pakistan \\ ${ }^{2}$ Department of Horticulture, PMAS-Arid Agriculture University, Rawalpindi, Pakistan
}

\begin{abstract}
The experiment under discussion was conducted at Fruit Crops Research Programme, of Horticulture Research Institute, National Agricultural Research Center, Islamabad, Pakistan (Latitude: $33.42^{\circ} \mathrm{N}$; Longitude: $73.08^{\circ} \mathrm{E}$; Elevation: $683 \mathrm{msl}$ ) during the years 2006-07. Effects of different mulching materials, such as, wheat straw, rice straw, transparent and black plastic mulch, were studied on the growth and performance of strawberry cultivar 'Chandler'. Maximum plant height $(17.70 \mathrm{~cm})$ was observed in rice straw mulch treatment. Whereas, black plastic mulch increased root length $(14.73 \mathrm{~cm})$, soil moisture (39.45), soil temperature (22.33), vegetative growth (Leaf area index 17.81, crown diameter $7.76 \mathrm{~cm}$ ), leaf chlorophyll (43.53), fruit yield (210.0) and total soluble solids (8.06). The rice and wheat straw mulch significantly ameliorated the fruit weight loss and spoilage percentage.
\end{abstract}

Key words: Moisture conservation, mulching, yield

\section{INTRODUCTION}

Mulching is the most important agricultural practice in strawberry crop, being exercised world-over. In addition to moisture conservation and temperature regulation, it avoids the contact of berries with soil and thus minimizes the chances of fruit rot, which adversely affects post-harvest life of the berries. Use of proper mulch is, thus, equally important for increasing shelf life (Sharma, 2002). Singh et al. (2005b) reported that, in northern plains of India, to get an early and high yield of the best quality strawberry could be planted in mid-September, if mulched with black polyethylene. Lareau and Lamarre (1993) compared straw mulch with black plastic mulch and concluded that black plastic mulch increased the yield, number and weight of crowns but decreased the fruit-size of tribute and tri-star strawberries.

Strawberry is known as the most delicious and refreshing fruit with high nutritive value (Cordenunsi et al., 2003) and high hexose/sucrose ratio (Nishizawa et al., 2005) to millions of people of the world. It is one of the most perishable fruits with short shelf life due to the susceptibility of berries to mechanical injury, water loss, decay and physiological deterioration (Nunes et al., 1995). Much research has been conducted and there were efforts of manipulating pre-harvest (Mukkun et al., 2001) and post harvest factors (Cordenunsi et al., 2005) that affect fruit quality in order to enhance strawberry shelf life. Its cultural practices are highly variable (Sharma, 2002). Superficial colour of berries determines the time of harvest, while fruit firmness is associated with shelf life. Recently, the determination of ascorbic acid has been of major interest (Aaby et al., 2005; Scalzo et al., 2005).

This study was conducted to measure the effect of mulching materials on yield, quality and shelf life of strawberry.

\section{MATERIALS AND METHODS}

Experiment was carried out during 2006-2007, at Fruit Crops Research Program, National Agricultural Research Center, Islamabad, Pakistan (Latitude: $33.42^{\circ} \mathrm{N}$; Longitude: $73.08^{\circ} \mathrm{E}$; Elevation: $683 \mathrm{msl}$ ). The present investigation was undertaken to determine the response of strawberry cultivar 'Chandler' to different mulching materials, such as wheat straw, rice straw, transparent plastic and black plastic mulch. Strawberry plants were grown in open field conditions, using different mulching materials as pre-harvest treatments. Field was ploughed up three times with disc harrow and leveled for better drainage. Farmyard manure was applied @ $20 \mathrm{t} \mathrm{ha}^{-1}$, ammonium sulfate@ $500 \mathrm{~kg} \mathrm{ha}^{-1}$, super phosphate @ $300 \mathrm{~kg} \mathrm{ha}^{-1}$ and potassium sulfate @ $150 \mathrm{~kg} \mathrm{ha}^{-1}$, prior to plough the soil. Raised beds of width $70 \mathrm{~cm}$ with height of $30 \mathrm{~cm}$ were prepared. 
Following treatments were applied during spring 2006 and 2007, under the field condition:

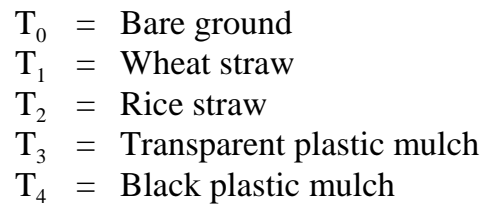

Periodic measurements of soil moisture from the same plot each day were taken from randomly selected plots for all treatments of a replication on an observation day. For soil moisture status, soil samples were taken in aluminum cans from 15-30 cm depth for each treatment and moisture percentage was determined by gravimetric method (Yasin et al., 1996). Root zone temperature was measured, using a bimetal thermometer (REOTEMP Instruments, San Diego, CA), that produced a direct measurement of soil temperature. Leaf area index was calculated, using the Leaf canopy analyzer 2000, LI-COR, Inc., 4421 (Welles and Norman, 1991). Leaf chlorophyll concentration was recorded by Spad-502 chlorophyll meter (Minolta Camera Co. Ltd., Osaka, Japan (Arenas et al., 2002).

\section{RESULTS AND DISCUSSION}

Available soil moisture: All mulched plots maintained higher soil moisture content at $30 \mathrm{~cm}$ depth from the top of the soil surface. Among the mulches used, the black plastic mulch had higher average soil moisture content, followed by transparent plastic mulch and wheat straw mulch had the least increase even that of the bare ground (Table 1). This occurs due to the fact that in the mulched soil, the evaporating soil moisture condenses on the mulch which serves as insulating substances and drips down again to the soil surface. Improvement of water infiltration and higher water retention resulting from mulches has also been reported by Swenson et al. (2004), Miller et al. (2002), Arriaga and Lowery (2003) and Hedau and Kumar (2002).

Soil temperature: Black plastic mulch treatment retained maximum $\left(22.3^{\circ} \mathrm{C}\right)$ soil temperature followed by transparent plastic mulch $\left(21.37^{\circ} \mathrm{C}\right)$. The potential of organic mulches (wheat and rice straw mulch) to retain soil temperature were lower than black plastic mulch (Table 1). Similar increase in soil temperature under black plastic mulch has been reported previously by Diaz-Perez et al. (2004). Minimum soil temperature was observed under rice straw mulch. These results are in agreement with those of Diaz-Perez et al. (2004), they found that mean soil temperature under black mulch was $9^{\circ} \mathrm{C}$ higher than that under organic mulch. Both wheat straw mulch and rice straw mulch resulted in significantly less soil moisture \% as compared to the bare ground.

Vegetative growth of plants: Mulching improved plant growth and development. Rice straw mulch produced the tallest plants. The highest leaf area index and crown size were recorded in black plastic mulch, followed by rice straw mulch. Root length was markedly higher in rice straw mulch and transparent mulch, followed by wheat straw mulch (Table 2 and 3). Increase in growth characteristics by mulching has also been reported by Agele et al. (2000), Singh et al. (2005a) in tomato and Dobbelaere et al. (2000), for strawberry. The present investigation established the fact that mulching improves the vegetative growth in strawberry. Similar results have also been reported by Moor et al. (2004).

Mulching inc reased the leaf chlorophyll content as recorded in black plastic mulch and rice straw mulch, followed by wheat straw mulch. This could be due to the fact that mulching also improved the Leaf Area Index (LAI) and retained by available soil moisture. The

Table 1: DMRT of treatment means showing the effect of mulching on soil temperature and moisture contents, (Data on soil temperature and moisture were collected weekly (Transplanting to final harvest). Each mean represents three replications and twenty observations recorded for each mean)

\begin{tabular}{|c|c|c|c|c|c|c|}
\hline \multirow[b]{2}{*}{ Treatments } & \multicolumn{3}{|c|}{ Soil temperature $\left({ }^{\circ} \mathrm{C}\right)$} & \multicolumn{3}{|c|}{ Soil moisture (\%) } \\
\hline & 2006 & 2007 & Mean & 2006 & 2007 & Mean \\
\hline Bare ground & 18.02 & 19.52 & $18.77^{\mathrm{c}}$ & $29.63^{\mathrm{i}}$ & $31.60^{\mathrm{h}}$ & $30.61^{\mathrm{e}}$ \\
\hline Wheat straw mulch & 15.7 & 16.3 & $16.00^{\mathrm{d}}$ & $34.33^{g}$ & $35.33^{f}$ & $34.83^{d}$ \\
\hline Rice straw mulch & 15.8 & 16.17 & $15.99^{d}$ & $35.79^{\mathrm{e}}$ & $36.80^{\mathrm{d}}$ & $36.30^{c}$ \\
\hline Transparent plastic mulch & 21.57 & 21.17 & $21.37^{\mathrm{b}}$ & $36.45^{d}$ & $37.42^{\mathrm{c}}$ & $36.94^{\mathrm{b}}$ \\
\hline Black plastic mulch & 22.33 & 22.33 & $22.33^{\mathrm{a}}$ & $38.71^{\mathrm{b}}$ & $39.45^{\mathrm{a}}$ & $39.08^{\mathrm{a}}$ \\
\hline LSD & 0.939 & & 0.664 & & 0.4402 & 0.3113 \\
\hline Mean of years & $18.68^{\mathrm{b}}$ & $19.1^{\mathrm{a}}$ & & $34.98^{\mathrm{b}}$ & $36.12^{\mathrm{a}}$ & \\
\hline Source & F value & & & F value & & \\
\hline Years & $6.5901^{*}$ & & & $27.5395 *$ & & \\
\hline Treatments & $168.0611^{* *}$ & & & $879.1486 * *$ & & \\
\hline Interaction & $2.4725^{\mathrm{NS}}$ & & & $5.0932 * *$ & & \\
\hline
\end{tabular}

Figures followed by different letters are significantly different at ( $\mathrm{p}>0.05)$, according to Duncan’s multiple range tests, NS : Non significant , * $>0.05$, $* * \mathrm{p}>0.01$ 
Sci. Technol. Dev., 35 (3): 117-122, 2016

Table 2: DMRT of treatment means showing the effect of mulching on vegetative growth of strawberry cv. Chandler. (Data on plant height, LAI and crown size were collected at final harvest. Each mean represents three replications and ten samples, collected for each mean.)

\begin{tabular}{|c|c|c|c|c|c|c|c|c|c|}
\hline \multirow[b]{2}{*}{ Treatments } & \multicolumn{3}{|c|}{ Plant height $(\mathrm{cm})$} & \multicolumn{3}{|c|}{ Leaf area index (LAI) } & \multicolumn{3}{|c|}{ Crown size $(\mathrm{cm})$} \\
\hline & 2006 & 2007 & Mean & 2006 & 2007 & Mean & 2006 & 2007 & Mean \\
\hline Bare ground & $16.70^{\mathrm{d}}$ & $16.02^{\mathrm{e}}$ & $16.36^{\mathrm{e}}$ & $10.68^{g}$ & $11.50^{f}$ & $11.09^{\mathrm{e}}$ & $0.97^{\mathrm{f}}$ & $0.97^{\mathrm{f}}$ & $0.97^{\mathrm{e}}$ \\
\hline Wheat straw mulch & $17.80^{\mathrm{ab}}$ & $17.60^{\mathrm{b}}$ & $17.70^{\mathrm{b}}$ & $15.43^{d}$ & $13.75^{\mathrm{e}}$ & $14.59^{\mathrm{c}}$ & $1.43^{\mathrm{c}}$ & $1.05^{\text {ef }}$ & $1.24^{\mathrm{c}}$ \\
\hline Rice straw mulch & $17.98^{\mathrm{a}}$ & $17.95^{\mathrm{a}}$ & $17.96^{\mathrm{a}}$ & $17.08^{\mathrm{b}}$ & $16.25^{\mathrm{c}}$ & $16.66^{\mathrm{b}}$ & $1.75^{\mathrm{b}}$ & $1.30^{\mathrm{d}}$ & $1.52^{\mathrm{b}}$ \\
\hline Transparent plastic mulch & $17.58^{\mathrm{b}}$ & $16.88^{\mathrm{cd}}$ & $17.23^{\mathrm{d}}$ & $13.93^{\mathrm{e}}$ & $11.65^{\mathrm{f}}$ & $12.79^{d}$ & $0.99^{\mathrm{f}}$ & $1.15^{\mathrm{e}}$ & $1.07^{\mathrm{d}}$ \\
\hline Black plastic mulch & $17.92^{\mathrm{a}}$ & $17.10^{\mathrm{c}}$ & $17.51^{\mathrm{c}}$ & $18.17^{\mathrm{a}}$ & $17.45^{\mathrm{ab}}$ & $17.81^{\mathrm{a}}$ & $1.87^{\mathrm{a}}$ & $1.65^{\mathrm{b}}$ & $1.76^{\mathrm{a}}$ \\
\hline LSD & 0.2485 & & 0.1757 & 0.7667 & & 0.5421 & 0.113 & & 0.07993 \\
\hline Mean of years & $17.6^{\mathrm{a}}$ & $17.11^{\mathrm{b}}$ & & $15.06^{\mathrm{a}}$ & $14.12^{\mathrm{b}}$ & & $1.40^{\mathrm{a}}$ & $1.22^{\mathrm{b}}$ & \\
\hline Source & F value & & & F value & & & F valu & & \\
\hline Years & 425746 & & & 23.623 & & & 52.054 & & \\
\hline Treatments & 104.46 & & & 218.77 & & & 153.05 & & \\
\hline Interaction & 8.4612 & & & 9.9435 & & & 23.059 & & \\
\hline
\end{tabular}

Table 3: DMRT of treatment means showing the effect of mulching on leaf chlorophyll contents, root length and fruit yield of strawberry cv. Chandler. (Data on leaf chlorophyll and fruit yield were collected periodically. Root length was collected at final harvest. Each mean represents three replications and ten observations, recorded for each mean)

\begin{tabular}{|c|c|c|c|c|c|c|c|c|c|}
\hline \multirow[b]{2}{*}{ Treatments } & \multicolumn{3}{|c|}{ Leaf chlorophyll } & \multicolumn{3}{|c|}{ Root length (cm) } & \multicolumn{3}{|c|}{ Fruit yield plant $^{-1}$ (g) } \\
\hline & 2006 & 2007 & Mean & 2006 & 2007 & Mean & 2006 & 2007 & Mean \\
\hline Bare ground & $39.22^{\mathrm{e}}$ & $41.00^{c}$ & $40.11^{\mathrm{d}}$ & 5.58 & 4.81 & $5.19^{d}$ & $180.3^{\mathrm{e}}$ & $169.8^{f}$ & $175.0^{\mathrm{e}}$ \\
\hline Wheat straw mulch & $41.33^{c}$ & $42.08^{\mathrm{b}}$ & $41.70^{\mathrm{b}}$ & 12.01 & 13.68 & $12.84^{\mathrm{b}}$ & $200.5^{b}$ & $203.0^{\mathrm{b}}$ & $201.8^{\mathrm{b}}$ \\
\hline Rice straw mulch & $42.33^{\mathrm{b}}$ & $44.38^{\mathrm{a}}$ & $43.35^{\mathrm{a}}$ & 11.8 & 10.15 & $10.97^{c}$ & $190.5^{c}$ & $185.0^{\mathrm{d}}$ & $187.8^{c}$ \\
\hline Transparent plastic mulch & $40.10^{\mathrm{d}}$ & $41.15^{c}$ & $40.63^{c}$ & 13.77 & 13.2 & $13.48^{\mathrm{ab}}$ & $183.5^{\mathrm{de}}$ & $180.3^{\mathrm{e}}$ & $181.9^{\mathrm{d}}$ \\
\hline Black plastic mulch & $42.58^{\mathrm{b}}$ & $44.47^{\mathrm{a}}$ & $43.53^{\mathrm{a}}$ & 15.36 & 14.1 & $14.73^{\mathrm{a}}$ & $210.5^{\mathrm{a}}$ & $209.5^{\mathrm{a}}$ & $210.0^{\mathrm{a}}$ \\
\hline LSD & 0.5538 & 0.3916 & NS & 1.606 & 3.732 & 2.639 & & & \\
\hline Mean of years & $41.11^{\mathrm{b}}$ & $42.62^{\mathrm{a}}$ & & 11.7 & 11.19 & & $193.05^{\circ}$ & $189.5^{\mathrm{b}}$ & \\
\hline Source & \multicolumn{3}{|l|}{ F value } & \multicolumn{3}{|c|}{$F$ value } & \multicolumn{3}{|l|}{ F value } \\
\hline Years & \multicolumn{3}{|c|}{$43.9597 * *$} & \multicolumn{3}{|c|}{$1.3083^{\mathrm{NS}}$} & \multicolumn{3}{|c|}{$22.0131^{* *}$} \\
\hline Treatments & \multicolumn{3}{|c|}{$133.4830 * *$} & \multicolumn{3}{|c|}{$46.4058 * *$} & \multicolumn{3}{|c|}{$252.6998 * *$} \\
\hline Interaction & \multicolumn{3}{|c|}{$4.5309 * *$} & \multicolumn{3}{|c|}{$1.3745^{\mathrm{NS}}$} & \multicolumn{3}{|c|}{$7.2887 * *$} \\
\hline
\end{tabular}

Figures followed by different letters are significantly different at ( $\mathrm{p}>0.05)$, according to Duncan’s multiple range tests, NS: Non significant $* \mathrm{p}>0.05$, $* * \mathrm{p}>0.01$

increased vegetative growth and Leaf Area Index (LAI) promote the solar radiation intercepted by the canopy and thus increase leaf chlorophyll contents as stated earlier by Kirnak et al. (2001). The leaf area index was significantly and positively correlated with chlorophyll content $\left(\mathrm{r}=0.721^{* *}\right)$ and fruit weight $\left(\mathrm{r}=0.712^{* *}\right)$ and available soil moisture contents.

Yield: Black plastic, transparent plastic and rice straw mulches produced higher weight of single fruit. However, the maximum fruit length was found in wheat straw mulch followed by black plastic mulch. The fruit width was not significantly affected by mulching. It was observed that mulching influenced yield significantly in strawberry, maximum yield (210.0 g) was obtained by the use of black plastic mulch, followed by wheat (201.8 g) and rice straw (187.8 g) mulch, respectively (Table 3), whereas, Table 4 describes the DMRT of treatment mean and shows the effect of mulching on fruit size and fruit weight of strawberry. Similar studies have been reported by Moor et al. (2004), that mulches had significant influence on yield and quality of strawberry.

Fruit quality: Rice straw mulch and transparent plastic mulch have no significant effect on ascorbic acid contents of strawberry fruit, in contrast wheat straw mulch and black plastic mulch showed decrease in ascorbic acid content. Ascorbic acid contents were lower in black plastic mulch treatment as compared to bare ground treatment. The relatively low content of ascorbic acid could be the causes of high temperature during ripening of the fruit. This is in line with studies conducted by Lee and Kadir (2000). Total Soluble Solids (TSS) were considerably affected by the mulching treatments. All types of mulches showed increase in TSS (Table 5). Moor et al. (2004) have described that application of organic mulch is better than plastic mulch as for production of better quality of strawberry. 
Sci. Technol. Dev., 35 (3): 117-122, 2016

Table 4: DMRT of treatment mean, showing the effect of mulching on fruit size and single fruit weight of strawberry cv. Chandler. (Data on fruit size were collected periodically. Each mean represents three replications and ten observations, recorded for each mean)

\begin{tabular}{|c|c|c|c|c|c|c|c|c|c|}
\hline \multirow[b]{2}{*}{ Treatments } & \multicolumn{3}{|c|}{ Single fruit weight (g) } & \multicolumn{3}{|c|}{ Fruit length $(\mathrm{cm})$} & \multicolumn{3}{|c|}{ Fruit width $(\mathrm{cm})$} \\
\hline & 2006 & 2007 & Mean & 2006 & 2007 & Mean & 2006 & 2007 & Mean \\
\hline Bare ground & $12.95^{\mathrm{c}}$ & $13.02^{\mathrm{c}}$ & $12.99^{\mathrm{c}}$ & $3.24 \mathrm{~d}$ & $3.08^{\mathrm{e}}$ & $3.16^{\mathrm{d}}$ & 2.83 & 2.81 & $2.82^{\mathrm{ab}}$ \\
\hline Wheat straw mulch & $15.02^{\mathrm{b}}$ & $14.85^{\mathrm{b}}$ & $14.94^{\mathrm{b}}$ & $3.45^{\mathrm{b}}$ & $3.55^{\mathrm{a}}$ & $3.50^{\mathrm{a}}$ & 2.75 & 2.83 & $2.79^{b}$ \\
\hline Rice straw mulch & $15.82^{\mathrm{a}}$ & $15.73^{\mathrm{a}}$ & $15.77^{\mathrm{a}}$ & $3.08 \mathrm{e}$ & $3.06 \mathrm{e}$ & $3.07 \mathrm{e}$ & 2.84 & 2.91 & $2.87^{\mathrm{a}}$ \\
\hline Transparent plastic mulch & $15.80^{\mathrm{a}}$ & $15.27^{\mathrm{ab}}$ & $15.54^{\mathrm{a}}$ & $3.26^{\mathrm{d}}$ & $3.21^{\mathrm{d}}$ & $3.23^{c}$ & 2.74 & 2.78 & $2.76^{\mathrm{b}}$ \\
\hline Black plastic mulch & $15.93^{\mathrm{a}}$ & $15.95^{\mathrm{a}}$ & $15.94^{\mathrm{a}}$ & $3.36^{c}$ & $3.48^{\mathrm{b}}$ & $3.42^{b}$ & 2.78 & 2.78 & $2.78^{\mathrm{b}}$ \\
\hline LSD & 0.6157 & 0.4354 & 0.06527 & 0.04615 & NS & 0.07297 & & & \\
\hline Mean of years & 15.11 & 14.97 & & 3.28 & 3.28 & & 2.79 & 2.82 & \\
\hline Source & $\mathrm{F}$ value & & & F value & & & F value & & \\
\hline Years & $0.8382^{N}$ & & & $0.0000^{\mathrm{NS}}$ & & & $1.4895^{\mathrm{NS}}$ & & \\
\hline Treatments & 65.3526 & & & 108.547 & & & $3.4561^{*}$ & & \\
\hline Interaction & $0.6306^{\mathrm{N}}$ & & & 10.7039" & & & $0.6652^{\mathrm{NS}}$ & & \\
\hline
\end{tabular}

Figures followed by different letters are significantly different at ( $>0.05$ ), according to Duncan's multiple range tests, NS : Non significant *p $>0.05$, ** $\mathrm{p}>0.01$

Table 5: DMRT of treatment means showing the effect of mulching on quality attributes of strawberry cv. Chandler. (Data on quality aspects were collected periodically after harvest. Each mean represents three replications and ten samples, collected for each mean)

\begin{tabular}{|c|c|c|c|c|c|c|c|c|c|}
\hline \multirow[b]{2}{*}{ Treatments } & \multicolumn{3}{|c|}{ Ascorbic acid (mg $100 \mathrm{~g}^{-1}$ ) } & \multicolumn{3}{|c|}{ Fruit firmness (lbs) } & \multicolumn{3}{|c|}{ TSS ( ${ }^{\circ}$ Brix $)$} \\
\hline & 2006 & 2007 & Mean & 2006 & 2007 & Mean & 2006 & 2007 & Mean \\
\hline Bare ground & $66.75^{\mathrm{a}}$ & $66.50^{\mathrm{a}}$ & $66.63^{\mathrm{a}}$ & $4.890^{\mathrm{ab}}$ & $4.900^{\mathrm{a}}$ & 4.9 & $7.33^{c}$ & $6.70^{\mathrm{d}}$ & $7.02^{\mathrm{c}}$ \\
\hline Wheat straw mulch & $54.00^{c}$ & $50.50^{c}$ & $52.25^{\mathrm{b}}$ & $4.900^{\mathrm{a}}$ & $4.860^{\mathrm{ab}}$ & 4.88 & $7.58^{\mathrm{bc}}$ & $7.63^{\mathrm{abc}}$ & $7.60^{\mathrm{b}}$ \\
\hline Rice straw mulch & $62.25^{\mathrm{b}}$ & $69.00^{\mathrm{a}}$ & $65.63^{\mathrm{a}}$ & $4.905^{\mathrm{a}}$ & $4.920^{\mathrm{a}}$ & 4.91 & $7.65^{\mathrm{abc}}$ & $8.28^{\mathrm{a}}$ & $7.96^{\mathrm{ab}}$ \\
\hline Transparent plastic mulch & $67.25^{\mathrm{a}}$ & $66.25^{\mathrm{a}}$ & $66.75^{\mathrm{a}}$ & $4.825^{\mathrm{b}}$ & $4.915^{\mathrm{a}}$ & 4.87 & $7.73^{\mathrm{abc}}$ & $8.23^{\mathrm{ab}}$ & $7.98^{\mathrm{ab}}$ \\
\hline Black plastic mulch & $53.50^{c}$ & $53.75^{c}$ & $53.63^{b}$ & $4.903^{\mathrm{a}}$ & $4.892^{\mathrm{ab}}$ & 4.9 & $7.90^{\mathrm{abc}}$ & $8.23^{\mathrm{ab}}$ & $8.06^{\mathrm{a}}$ \\
\hline LSD & 3.32 & 2.348 & 0.06527 & NS & 0.6 & 0.4242 & & & \\
\hline Mean of years & 60.75 & 61.2 & & 4.88 & 4.9 & & 7.64 & 7.81 & \\
\hline Source & F value & & & F value & & & F value & & \\
\hline Years & $1.4211^{\mathrm{NS}}$ & & & $0.6747^{\mathrm{NS}}$ & & & $1.5685^{\mathrm{NS}}$ & & \\
\hline Treatments & $83.8816 * *$ & & & $1.4425^{\mathrm{NS}}$ & & & $8.9585 * *$ & & \\
\hline Interaction & $5.5966 * *$ & & & $3.0915 *$ & & & $2.9215 *$ & & \\
\hline
\end{tabular}

Figures followed by different letters are significantly different at ( $\mathrm{p}>0.05)$, according to Duncan’s multiple range tests, NS: Non significant * $\mathrm{p}>0.05$, $* * \mathrm{p}>0.01$

Table 6: DMRT of treatment means showing the effect of mulching on shelf life of strawberry cv. Chandler. (Data on shelf life aspects were recorded after harvest (periodically). Each mean represents three replications and five samples, collected for each mean)

\begin{tabular}{|c|c|c|c|c|c|c|c|c|c|}
\hline \multirow[b]{2}{*}{ Treatments } & \multicolumn{3}{|c|}{ Weight loss (\%) } & \multicolumn{3}{|c|}{ Spoilage (\%) } & \multicolumn{3}{|c|}{ Shelf life (days) } \\
\hline & 2006 & 2007 & Mean & 2006 & 2007 & Mean & 2006 & 2007 & Mean \\
\hline Bare ground & 46.25 & 47.25 & $46.75^{\mathrm{a}}$ & $54.50^{\mathrm{b}}$ & $56.25^{\mathrm{ab}}$ & $55.38^{\mathrm{a}}$ & 2.5 & 2 & $2.3^{\mathrm{b}}$ \\
\hline Wheat straw mulch & 30.25 & 33 & $31.63^{d}$ & $45.00^{d}$ & $50.00^{c}$ & $47.50^{c}$ & 3 & 2.5 & $2.8^{\mathrm{a}}$ \\
\hline Rice straw mulch & 31.25 & 33.25 & $32.25^{\mathrm{d}}$ & $45.00^{d}$ & $43.00^{\mathrm{d}}$ & $44.00^{d}$ & 2 & 2 & $2.0^{\mathrm{b}}$ \\
\hline Transparent plastic mulch & 40 & 40.25 & $40.13^{b}$ & $51.00^{c}$ & $59.25^{\mathrm{a}}$ & $55.13^{\mathrm{ab}}$ & 2 & 2 & $2.0^{\mathrm{b}}$ \\
\hline Black plastic mulch & 37 & 39.5 & $38.25^{c}$ & $54.50^{\mathrm{b}}$ & $56.25^{\mathrm{ab}}$ & $55.38^{\mathrm{a}}$ & 3 & 2.8 & $2.9^{\mathrm{a}}$ \\
\hline LSD & NS & 1.138 & 3.082 & 2.179 & NS & 0.3263 & & & \\
\hline Mean of years & $36.95^{\mathrm{b}}$ & $38.65^{\mathrm{a}}$ & & $49.15^{\mathrm{b}}$ & $52.85^{\mathrm{a}}$ & & $2.5^{\mathrm{a}}$ & $2.3^{\mathrm{b}}$ & \\
\hline Source & \multicolumn{3}{|c|}{ F value } & \multicolumn{3}{|l|}{ F value } & \multicolumn{3}{|c|}{ F value } \\
\hline Years & \multicolumn{3}{|c|}{$27.5238^{* *}$} & \multicolumn{3}{|c|}{$48.0351^{* *}$} & \multicolumn{3}{|c|}{$10.7143^{*}$} \\
\hline Treatments & \multicolumn{3}{|c|}{$254.2089 * *$} & \multicolumn{3}{|c|}{$45.5332^{* *}$} & \multicolumn{3}{|c|}{$13.7500 * *$} \\
\hline Interaction & \multicolumn{3}{|c|}{$1.8185^{\mathrm{NS}}$} & \multicolumn{3}{|c|}{$6.9514^{* *}$} & \multicolumn{3}{|c|}{$1.2500^{\mathrm{NS}}$} \\
\hline
\end{tabular}

Figures followed by different letters are significantly different at ( $\mathrm{p}>0.05)$, according to Duncan’s multiple range tests. NS: Non significant * $>0.05$, $* * \mathrm{p}>0.01$

Shelf life: Organic mulching (wheat and rice straw mulch) reduced weight loss and fruit spoilage in strawberry fruits and improved the shelf life. Minimum fruit spoilage and weight loss was recorded in wheat straw mulch and rice straw mulch, respectively. The black and transparent plastic mulch increased the weight loss in strawberry cultivar 'Chandler'. The increase in weight loss in strawberry fruit produced under plastic mulches could be due to high soil temperature which affects the growth inhibitory hormone and ethylene stress might be increased under high soil temperature produced by these mulches (Table 6). As per statistical analysis wheat straw and black mulch produced strawberry with maximum shelf life. Sharma and Agrawal (2004) also stated that mulching with black plastic and erection of row covers in winter is very beneficial for better growth and production of strawberry in sub-tropical conditions. 
Although, interaction of treatments and year were highly significant regarding $\mathrm{pH}$ of fruit, there was not any consistency among treatments in both years.

\section{CONCLUSION}

It can be inferred that application of black plastic mulch and rice straw mulch could be highly effective in maintaining soil moisture content in strawberry fields. The black plastic mulch retained higher soil moisture and temperature than the rest of the treatments. Maximum plant height and root length was observed in rice straw mulch treatment, whereas, black plastic mulch resulted in increased Leaf Area Index (LAI), crown size, leaf chlorophyll and total fruit yield. The rice and wheat straw mulch significantly ameliorated the post harvest fruit weight loss and spoilage percentage. Black plastic mulch significantly reduced the ascorbic acid content but increased the Total Soluble Solids (TSS).

\section{REFERENCES}

Aaby, K., G. Skrede and R.E. Wrolstad, 2005. Phenolic composition and antioxidant activities in flesh and achenes of strawberries (Fragaria ananassa). J. Agric. Food Chem., 53: 4032-4040.

Agele, S.O., G.O. Iremiren and S.O. Ojeniyi, 2000. Effects of tillage and mulching on the growth, development and yield of late-season tomato (Lycopersicon esculentum L.) in the humid south of Nigeria. J. Agric. Sci., 134: 55-59.

Arenas, M., C.S. Vavrina, J.A. Cornell, E.A. Hanlon and G.J. Hochmuth, 2002. Coir as an alternative to peat in media for tomato transplant production. HortScience, 37: 309-312.

Arriaga, F.J. and B. Lowery, 2003. Soil physical properties and crop productivity of an eroded soil amended with cattle manure. Soil Sci., 168: 888-899.

Cordenunsi, B.R., J.D. Nascimento and F.M. Lajolo, 2003. Physico-chemical changes related to quality of five strawberry fruit cultivars during cool-storage. Food Chem., 83: 167-173.

Cordenunsi, B.R., M.I. Genovese, J.R.O. do Nascimento, N.M.A. Hassimotto, R.J. dos Santos and F.M. Lajolo, 2005. Effects of temperature on the chemical composition and antioxidant activity of three strawberry cultivars. Food Chem., 91: 113-121.

Diaz-Perez, J.C., D. Granberry, D. Bertrand and D. Giddings, 2004. Tomato plant growth during establishment as affected by root zone temperature under colored mulches. Acta Hortic., 631: 119-124.
Dobbelaere, S., A. Croonenboghs, A. Thys, D. Ptacek and J. Vanderleyden et al., 2001. Responses of agronomically important crops to inoculation with Azospirillum. Aust. J. Plant Physiol., 28: 871-879.

Hedau, N.K. and M. Kumar, 2002. Effect of different mulches on yield, plant height, nitrogen uptake, weed control, soil moisture and economics of tomato cultivation. Prog. Hortic., 34: 208-210.

Kirnak, H., C. Kaya, D. Higgs and S. Gercek, 2001. A long-term experiment to study the role of mulches in the physiology and macro-nutrition of strawberry grown under water stress. Aust. J. Agric. Res., 52: 937-943.

Lareau, M.J. and M. Lamarre, 1993. Late planting of strawberry using bare root or plug plants. Acta Hortic., 348: 245-248.

Lee, S.K. and A.A. Kader, 2000. Preharvest and postharvest factors influencing vitamin $C$ content of horticultural crops. Postharvest Biol. Technol., 20: 207-220.

Miller, J.J., N.J. Sweetland and C. Chang, 2002. Hydrological properties of a clay loam soil after long-term cattle manure application. J. Environ. Qual., 31: 989-996.

Moor, U., K. Karp and P. Poldma, 2004. Effect of mulching and fertilization on the quality of strawberries. Agric. Food Sci., 13: 256-267.

Mukkun, L., Z. Singh and D. Phillips, 2001. Nitrogen nutrition affects fruit firmness, quality and shelf life of strawberry. Acta Hortic., 553: 69-71.

Nishizawa, T., S. Nagasawa, Y. Mori, Y. Kondo, Y. Sasaki, J.B. Retamales and A. Lavin, 2005. Characteristics of soluble sugar accumulation in commercially grown Fragaria chiloensis. HortScience, 40: 1647-1648.

Nunes, M.C.N., J.K. Brecht, A.M.M.B. Morais and S.A. Sargent, 1995. Physical and chemical quality characteristics of strawberries after storage are reduced by a short delay to cooling. Postharvest Biol. Technol., 6: 17-28.

Scalzo, J., A. Politi, N. Pellegrini, B. Mezzetti and M. Battino, 2005. Plant genotype affects total antioxidant capacity and phenolic contents in fruit. Nutrition, 21: 207-213.

Sharma, H.G. and N. Agrawal, 2004. Effect of different color mulches on the growth and yield of tomato under drip irrigation. Plant-Arch., 4: 93-99.

Sharma, R.R., 2002. Growing Strawberries. International Book Distributing Co., India, ISBN-13: 978-8185860893, Pages: 164.

Singh, B., M. Kumar and G.C. Singh, 2005a. Effect of different plastic mulches on growth and yield of winter tomato. Indian J. Horticult., 62: 200-202. 
Singh, R., R.R. Sharma and R.K. Jain, 2005b. Planting time and mulching influenced vegetative and reproductive traits in strawberry (Fragaria $\mathrm{x}$ ananassa Duch.) in India. Fruits, 60: 395-403

Swenson, J.A., S.A. Walters and S.K. Chong, 2004. Influence of tillage and mulching systems on soil water and tomato fruit yield and quality. J. Veg. Crop Prod., 10: 81-95.

Welles, J.M. and J.M. Norman, 1991. Instrument for indirect measurement of canopy architecture. Agron. J., 83: 818-825.
Yasin, M., M.T. Rashid and A. Jabbar, 1996. Effect of 4-cholorophenoxy acetic acid on tomato yield under high temperature conditions: Management of heat, moisture and other physical stress factors of tomatoes and chilies in Pakistan. Proceedings of the Phase I Final Workshop of South Asian Vegetable Research Network, January 23-28, 1996, Kathmandu, Nepal. 